\title{
A stability result using the matrix norm to bound the permanent
}

\author{
Ross Berkowitz* Pat Devlin ${ }^{\dagger}$
}

June 23, 2016

\begin{abstract}
We prove a stability version of a general result that bounds the permanent of a matrix in terms of its operator norm. More specifically, suppose $A$ is an $n \times n$ matrix over $\mathbb{C}$ (resp. $\mathbb{R}$ ), and let $\mathcal{P}$ denote the set of $n \times n$ matrices over $\mathbb{C}$ (resp. $\mathbb{R}$ ) that can be written as a permutation matrix times a unitary diagonal matrix. Then it is known that the permanent of $A$ satisfies $|\operatorname{per}(A)| \leq\|A\|_{2}^{n}$ with equality iff $A /\|A\|_{2} \in \mathcal{P}$ (where $\|A\|_{2}$ is the operator 2-norm of $A$ ). We show a stability version of this result asserting that unless $A$ is very close (in a particular sense) to one of these extremal matrices, its permanent is exponentially smaller (as a function of $n$ ) than $\|A\|_{2}^{n}$. In particular, for any fixed $\alpha, \beta>0$, we show that $|\operatorname{per}(A)|$ is exponentially smaller than $\|A\|_{2}^{n}$ unless all but at most $\alpha n$ rows contain entries of modulus at least $\|A\|_{2}(1-\beta)$.
\end{abstract}

\section{Introduction}

The permanent of an $n \times n$ matrix, $A$, has long been an important quantity in combinatorics and computer science, and more recently it has also had applications to physics and linear-optical quantum computing. It is defined as

$$
\operatorname{per}(A):=\sum_{\sigma \in S_{n}} \prod_{i=1}^{n} a_{i, \sigma(i)},
$$

where $S_{n}$ denotes the set of permutations of $[n]=\{1,2, \ldots, n\}$. For instance, if $A$ only has entries in $\{0,1\} \subseteq \mathbb{R}$, then the permanent counts the number of perfect matchings in the bipartite graph whose bipartite adjacency matrix is $A$.

The definition of the permanent is of course reminiscent of that for the determinant; however, whereas the determinant is rich in algebraic and geometric meaning, the more combinatorial permanent is notoriously difficult to understand. For example, computing $\operatorname{per}(A)$ even for $\{0,1\}$-matrices is the prototypical \#P-complete problem (Valiant [13]).

On the other hand, the operator 2-norm (also called the operator norm) of a matrix is a particularly nice parameter. For an $n \times n$ matrix $A$ with entries in $\mathbb{C}$, it is defined as

$$
\|A\|_{2}=\sup _{\|\vec{x}\|_{2} \leq 1, \vec{x} \in \mathbb{C}^{n}}\|A \vec{x}\|_{2}
$$

where $\|\vec{v}\|_{p}$ is the usual $l_{p}$ norm (i.e., $\|\vec{v}\|_{p}^{p}=\sum_{i}\left|v_{i}\right|^{p}$ for $p \in(0, \infty)$, and $\left.\|\vec{v}\|_{\infty}=\max \left|v_{i}\right|\right)$. The operator norm of a matrix has the advantages of being both algebraically and analytically well-behaved as well as computationally easy to determine (as this amounts to finding the largest singular value of $A$ ).

\footnotetext{
${ }^{*}$ Rutgers University. Email: rkb73@math.rutgers.edu

${ }^{\dagger}$ Rutgers University. Email: prd41@math.rutgers.edu. Supported by NSF grant DMS1501962.
} 
Considering how differently behaved the permanent and operator norm are, it is perhaps strange to think that there would be much of a connection between them. Nonetheless, they are related by the following extremal result, which is due to Gurvits [6] (see also [1, 2]).

Theorem 1. Suppose $A$ is an $n \times n$ matrix over $\mathbb{C}($ resp. $\mathbb{R})$, and let $\mathcal{P}$ denote the set of $n \times n$ matrices over $\mathbb{C}$ (resp. $\mathbb{R})$ that can be written as a permutation matrix times a unitary diagonal matrix. Then $|\operatorname{per}(A)| \leq\|A\|_{2}^{n}$ with equality iff $A$ is a scalar multiple of a matrix in $\mathcal{P}$.

Note that this extremal set $\mathcal{P}$ is simply the set of matrices with exactly $n$ non-zero entries, each having modulus 1 , and no two of which are in the same row or column. Such a matrix $P \in \mathcal{P}$ has $\|P\|_{2}=|\operatorname{per}(P)|=1$ and satisfies

$$
\|A P\|_{2}=\|P A\|_{2}=\|A\|_{2}, \quad \text { and } \quad|\operatorname{per}(A P)|=|\operatorname{per}(P A)|=|\operatorname{per}(A)|
$$

for all matrices $A$ (which is equivalent to membership in $\mathcal{P}$ ). Moreover, $\mathcal{P}$ is a subgroup of the group of unitary matrices, and as a set, it has a very tractable topological structure.

Motivated by algorithmic questions related to approximating the permanent, Aaronson and Hance [1] asked whether one could prove a stability version of Theorem [1]

Question A: If $|\operatorname{per}(A)|$ is close to $\|A\|_{2}^{n}$, must $A /\|A\|_{2}$ be 'close' to a matrix in $\mathcal{P}$ ?

A somewhat more concrete version was suggested by Aaronson and Nguyen [2]:

Question B: Characterize $n \times n$ matrices $A$ such that $\|A\|_{2} \leq 1$ and there exists a constant $C>0$ such that $|\operatorname{per}(A)| \geq n^{-C}$.

Using techniques of inverse Littlewood-Offord theory, Aaronson and Nguyen gave a substantial answer to an analogous question under the (stronger) assumptions that $A$ is orthogonal and that the intersection of the hypercube $\{ \pm 1\}^{n}$ with its image under $A$ is large. They also proved something like (actually slightly stronger than) our results below for stochastic matrices. Further results in the direction of Question B were given by Nguyen [10.

The two main results of the present paper are Theorems 2 and 3 below. The first provides a positive answer to Question A for matrices over $\mathbb{C}$ (or $\mathbb{R}$ ), and the second is a more refined result that (depending on your philosophical views) at least partially addresses Question B for matrices over $\mathbb{R}$. More specifically, we bound $\operatorname{per}(A)$ in terms of the following easily computed parameters.

Definition: Let $A$ be a matrix with rows $r_{1}, r_{2}, \ldots, r_{n}$, and $p \in \mathbb{R} \cup\{\infty\}$. Then the parameter $h_{p}(A)$ is defined as $h_{p}(A)=h_{p}=\frac{1}{n} \sum_{i}\left\|r_{i}\right\|_{p}$.

We will only consider $h_{\infty}$ and $h_{2}$. First note $0 \leq h_{\infty}(A) \leq h_{2}(A) \leq\|A\|_{2}$. Moreover, it is easy to show $h_{2}(A)=\|A\|_{2}$ iff $A /\|A\|_{2}$ is a unitary matrix, and $h_{\infty}(A)=\|A\|_{2}$ iff $A /\|A\|_{2}$ is in $\mathcal{P}$. Thus, in some sense, the quantity $1-h_{2}(A) /\|A\|_{2} \in[0,1]$ measures how close $A /\|A\|_{2}$ is to being unitary, and $1-h_{\infty}(A) /\|A\|_{2} \in[0,1]$ measures how close $A /\|A\|_{2}$ is to being in $\mathcal{P}$. Broadly speaking, $h_{\infty} /\|A\|_{2}$ is close to 1 precisely when most of the rows of $A$ each have one entry of modulus close to $\|A\|_{2}$ and all the other entries in that row are close to 0 .

Before stating the first of our main results, notice that in addressing either of the above questions, we lose no generality in assuming $\|A\|_{2} \leq 1$, since Question $\mathrm{A}$ is invariant under scaling. However, to facilitate any application of our results, we state them in the "more general" case that $\|A\|_{2} \leq T$. 
Theorem 2. Let $A$ be an $n \times n$ matrix over $\mathbb{C}$ and $\|A\|_{2} \leq T \neq 0$. Then

$$
\begin{aligned}
& \text { (i) }|\operatorname{per}(A)| \leq 2 T^{n} \exp \left[-3 n\left(1-\frac{\sqrt{\pi}}{2} h_{2} / T-\left(1-\frac{\sqrt{\pi}}{2}\right) h_{\infty} / T\right)^{2} / 100\right] \text {, } \\
& \text { (ii) }|\operatorname{per}(A)| \leq 2 T^{n} \exp \left[-n\left(1-h_{\infty} / T\right)^{2} / 10^{5}\right] \text {. }
\end{aligned}
$$

As discussed above, this provides a positive answer to Question A by viewing $h_{\infty}$ (and to a lesser extent $h_{2}$ ) as a proxy for 'closeness' of a matrix $A$ to those in $\mathcal{P}$. As an easy corollary, if $\alpha, \beta \geq 0$ satisfy $|\operatorname{per}(A)| \geq 2 T^{n} \exp \left[-n \alpha^{2} \beta^{2} / 10^{5}\right]$, then all but at most $\alpha n$ of the rows of $A$ contain an entry whose modulus is at least $T(1-\beta)$. And since the $l_{2}$ norm of any row of $A$ is at most $\|A\|_{2}$, no entry of $A$ can have modulus larger than $T$. Thus, entries of modulus $T(1-\beta)$ are nearly as large as possible. Moreover, if a row (or column) has an entry with very large modulus, then the remaining entries must have very small moduli (again since its $l_{2}$ norm is at most $\|A\|_{2}$ ). Thus, this theorem also provides a qualitative stability result stating that matrices with large permanent must have many very large entries, and a row (or column) containing a large entry must have all its other entries small.

Note that Theorem 2 is only useful for values of $h_{\infty} / T$ that are not very close to 1 - namely when $1-h_{\infty} / T \gg n^{-1 / 2}$. Although this does well in many cases, we believe that for large values of $h_{\infty} / T$, it is not optimal. For comparison, if $A$ is $\delta$ times the identity matrix, and $\delta \approx 1$, then $|\operatorname{per}(A)| \approx e^{-n(1-\delta)}=e^{-n\left(1-h_{\infty}\right)}$, and we conjecture that this is essentially tight.

Conjecture 1. There is some constant $C>0$ and some polynomial $f(n)$ such that the following holds. If $A$ is an $n \times n$ matrix with complex entries and $\|A\|_{2} \leq 1$, then $|\operatorname{per}(A)| \leq f(n) e^{-C n\left(1-h_{\infty}\right)}$.

As a step in this direction, we are able to prove the following, which better addresses Question B for matrices over $\mathbb{R}$.

Theorem 3. Let $A$ be an $n \times n$ matrix over $\mathbb{R}$ and $\|A\|_{2} \leq T \neq 0$. Then

$$
|\operatorname{per}(A)| \leq T^{n}(n+6) \exp \left[\frac{-\sqrt{n\left(1-h_{\infty} / T\right)}}{400}\right]
$$

As with Theorem 2, a result like Theorem 3 that involves $h_{2}$ is also possible, and it essentially falls out of our proof directly. Theorem 3 is an improvement over Theorem 2 when $n^{-1 / 3} \gg 1-h_{\infty} / T$ and gives a meaningful bound provided $1-h_{\infty} / T \gg \log (n)^{2} / n$. Although this yields a quantitatively better understanding for matrices over $\mathbb{R}$, we cannot shake the belief that neither of our main results (i.e., Theorems 2 and 3) is best possible, and we discuss this further in Section [5].

\section{Structure of paper}

The paper is devoted to proving Theorems 2 and 3, which goes roughly as follows. First, we appeal to a result of Glynn [5] that allows us to convert the problem of estimating the permanent into a problem about estimating the expected value of a certain random variable (Section 2). We then use standard probabilistic tools to show certain concentration results for the random variable of interest, which in turn yield the estimates needed for our results. This is done for the complex-valued case in Section 3 , which proves Theorem 2, In Section 4, we consider the real-valued case, where we analyze the corresponding random variable more carefully to obtain Theorem 3 . We conclude in Section 5 with several open questions and conjectures, as well as a discussion of Question B.

\section{Definitions and set-up with random variables}

We first need to use an observation due to Glynn [5] whereby the permanent of a matrix is expressed as the expectation of a certain random variable. We will work over the field $\mathbb{K}$, which will either be $\mathbb{R}$ or $\mathbb{C}$. 
Given an $n \times n$ matrix $A$ over $\mathbb{K}$ and $x \in \mathbb{K}^{n}$, set $y=A x$, and define the Glynn estimator of $A$ at $x$ to be

$$
\operatorname{Gly}_{x}(A)=\prod_{i=1}^{n} \bar{x}_{i} \times \prod_{i=1}^{n} y_{i}
$$

where $\bar{z}$ denotes the complex conjugate of $z$. Let $X \in \mathbb{K}^{n}$ be the random variable whose coordinates are independently selected uniformly on $|z|=1$, and let $Y=A X$ (note: if $\mathbb{K}=\mathbb{C}$, then each coordinate of $X$ is distributed continuously over the unit circle, whereas if $\mathbb{K}=\mathbb{R}$, then $X$ is chosen uniformly from the discrete set $\left.\{-1,1\}^{n}\right)$. Then

$$
\operatorname{per}(A)=\mathbb{E}\left[G l y_{X}(A)\right]=\mathbb{E}\left[\prod_{i=1}^{n} \bar{X}_{i} Y_{i}\right]
$$

obtained simply by expanding out the product in the Glynn estimator and using the fact that the $X_{i}$ are independent with mean 0 and variance 1 (see the original proof due to Glynn [5] or also [6, 1, 2]). Therefore, by convexity (which we are about to use twice), we have

$$
|\operatorname{per}(A)| \leq \mathbb{E}\left[\prod_{i=1}^{n}\left|\bar{X}_{i} Y_{i}\right|\right]=\mathbb{E}\left[\prod_{i=1}^{n}\left|Y_{i}\right|\right] \leq \mathbb{E}\left[\left(\frac{1}{n} \sum_{i=1}^{n}\left|Y_{i}\right|\right)^{n}\right]=\mathbb{E}\left[\left(\frac{\|A X\|_{1}}{n}\right)^{n}\right] .
$$

Note that from here, we could say (by Cauchy-Schwartz)

$$
\frac{\|A X\|_{1}}{n} \leq \frac{\|A X\|_{2}}{\sqrt{n}}=\frac{\|A X\|_{2}}{\|X\|_{2}} \leq\|A\|_{2}
$$

thus obtaining the inequality $|\operatorname{per}(A)| \leq\|A\|_{2}^{n}$ of Theorem 1 (the equality case follows by considering equality in the above estimates).

\section{Specializing to norm at most 1}

Note that to prove our results, it suffices to prove them for the case $\|A\|_{2} \leq 1$. This is because otherwise, we could simply scale the matrix by some $\alpha$ to have norm at most 1 , and because $\operatorname{per}(A)=\alpha^{n} \operatorname{per}(A / \alpha)$, our results would follow. As such, we will henceforth assume $\|A\|_{2} \leq 1$ (explicitly making note of when we do), but this choice is simply for notational ease. We remark that the set-up thus far has also been employed in several other papers [6, 1, 2]; however, the remainder of this paper deviates from the previous literature.

\section{Proof of Theorem $2(\mathbb{K}=\mathbb{C})$}

In the setting where $\|A\|_{2} \leq 1$, the permanent is always bounded above by 1 (as shown above), and we want to conclude that under certain conditions, it must be (exponentially) small. We know (since $0 \leq\|A X\|_{1} / n \leq\|A\|_{2} \leq 1$ ) that for all $\varepsilon \geq 0$ and all $\tilde{\mu} \geq 0$,

$$
|\operatorname{per}(A)| \leq \mathbb{E}\left[\left(\frac{\|A X\|_{1}}{n}\right)^{n}\right] \leq(\tilde{\mu} / n+\varepsilon)^{n}+\mathbb{P}\left(\|A X\|_{1} \geq \tilde{\mu}+\varepsilon n\right) .
$$

We will pick $\tilde{\mu}$ suitably small with $\tilde{\mu} \geq \mathbb{E}\left[\|A X\|_{1}\right]$ and then argue that $\|A X\|_{1}$ is tightly concentrated about its mean, which will complete the proof.

\section{The mean of $\|A X\|_{1}$}

We appeal to a theorem of König, Schütt, and Tomczak-Jaegermann [8], which is a variant of Khintchine's inequality conveniently well-suited for our situation (in fact, $X$ was chosen in part so that we could apply this result directly). 
Theorem 4 (König et al. 8], 1999). Let $\mathbb{K}$ be $\mathbb{R}$ or $\mathbb{C}$. Suppose $\vec{a}=\left(a_{1}, \ldots, a_{n}\right) \in \mathbb{K}^{n}$ is fixed, and suppose each coordinate of $\xi \in \mathbb{K}^{n}$ is independently distributed uniformly on $|z|=1$. Then

$$
\left|\mathbb{E}\left[\left|\sum_{i} a_{i} \xi_{i}\right|\right]-\Lambda_{\mathbb{K}}\|\vec{a}\|_{2}\right| \leq\left(1-\Lambda_{\mathbb{K}}\right)\|\vec{a}\|_{\infty},
$$

where $\Lambda_{\mathbb{R}}=\sqrt{2 / \pi}$ and $\Lambda_{\mathbb{C}}=\sqrt{\pi} / 2$.

Applying this to each row of $A$ (and using linearity of expectation) gives

Proposition 5. With $A$ and $X \in \mathbb{C}^{n}$ as in Section Q, we have

$$
\mathbb{E}\left[\|A X\|_{1} / n\right] \leq \frac{1}{n} \sum_{i=1}^{n}\left[\sqrt{\pi} / 2\left\|r_{i}\right\|_{2}+(1-\sqrt{\pi} / 2)\left\|r_{i}\right\|_{\infty}\right]=\frac{\sqrt{\pi}}{2} h_{2}(A)+\left(1-\frac{\sqrt{\pi}}{2}\right) h_{\infty}(A) .
$$

\section{Concentration about mean}

To show concentration of $\|A X\|_{1}$ about its mean, we use a very general and useful result of Talagrand (a form of "Talagrand's inequality"), which can be found in chapter 1 of his book [9].

Theorem 6 (Talagrand [9], 1991). Suppose $f: \mathbb{R}^{n} \rightarrow \mathbb{R}$ is such that $|f(x)-f(y)| \leq \sigma\|x-y\|_{2}$ for all $x, y \in \mathbb{R}^{n}$, and define the random variable $F=f\left(\xi_{1}, \xi_{2}, \ldots, \xi_{n}\right)$, where the $\xi_{i}$ are independent standard normal random variables. Then for all $t \geq 0$,

$$
\mathbb{P}(F>\mathbb{E}[F]+t) \leq e^{-2 t^{2} /(\pi \sigma)^{2}} .
$$

We apply this result to our setting by way of a now standard trick that expresses our random variable of interest as a function of standard Gaussians. In fact, this trick is even discussed in [9], so we could have saved a few lines of the following argument by simply citing a "more applicable" version of Theorem 6 (i.e., one for which this trick has already been incorporated); however, the trick so nicely captures the usefulness of Theorem [ 6 that we thought it worth recalling here.

Proposition 7. Suppose $\|A\|_{2} \leq 1$, and let $X \in \mathbb{C}^{n}$ be as in Section Q. Then for all $t \geq 0$,

$$
\mathbb{P}\left(\|A X\|_{1}>\mathbb{E}\left[\|A X\|_{1}\right]+t n\right) \leq e^{-n t^{2} / \pi^{3}} .
$$

Proof. To make use of Theorem [, we need to define a suitable $f: \mathbb{R}^{n} \rightarrow \mathbb{R}$, which we do in pieces. First define $\Phi: \mathbb{R} \rightarrow \mathbb{R}$ via

$$
\Phi(u)=\frac{1}{\sqrt{2 \pi}} \int_{-\infty}^{u} e^{-x^{2} / 2} d x
$$

which is the probability that a standard Gaussian is at most $u$. Then define $g: \mathbb{R}^{n} \rightarrow \mathbb{C}^{n}$ as

$$
g\left(x_{1}, \ldots, x_{n}\right)=\left(\begin{array}{c}
e^{2 \pi i \Phi\left(x_{1}\right)} \\
e^{2 \pi i \Phi\left(x_{2}\right)} \\
\vdots \\
e^{2 \pi i \Phi\left(x_{n}\right)}
\end{array}\right)
$$

and, finally, set $f(x)=\|A g(x)\|_{1}$.

Notice that if $\xi_{1}, \xi_{2}, \ldots, \xi_{n}$ are independently sampled from the standard normal distribution, then each $\Phi\left(\xi_{i}\right)$ is distributed uniformly on $[0,1]$. Therefore $g\left(\xi_{1}, \ldots, \xi_{n}\right)$ has the same distribution as $X$, and so $F:=f\left(\xi_{1}, \ldots, \xi_{n}\right)$ has the same distribution as $\|A X\|_{1}$. 
Now let $x, y \in \mathbb{R}^{n}$ be arbitrary. Then we have

$$
\begin{aligned}
|f(x)-f(y)| & =\left|\|A g(x)\|_{1}-\|A g(y)\|_{1}\right| \leq\|A g(x)-A g(y)\|_{1} \leq \sqrt{n}\|A(g(x)-g(y))\|_{2} \\
& \leq \sqrt{n}\|A\|_{2}\|g(x)-g(y)\|_{2} \leq \sqrt{n}\|g(x)-g(y)\|_{2} .
\end{aligned}
$$

Using the fact that $\left|e^{i \alpha}-1\right| \leq|\alpha|$ for all $\alpha \in \mathbb{R}$, we further bound the above by

$$
\begin{aligned}
\|g(x)-g(y)\|_{2}^{2} & =\sum_{j=1}^{n}\left|e^{2 \pi i \Phi\left(x_{j}\right)}-e^{2 \pi i \Phi\left(y_{j}\right)}\right|^{2}=\sum_{j=1}^{n}\left|e^{2 \pi i\left(\Phi\left(x_{j}\right)-\Phi\left(y_{j}\right)\right)}-1\right|^{2} \\
& \leq(2 \pi)^{2} \sum_{j=1}^{n}\left|\Phi\left(x_{j}\right)-\Phi\left(y_{j}\right)\right|^{2} \leq 2 \pi \sum_{j=1}^{n}\left|x_{j}-y_{j}\right|^{2}=2 \pi\|x-y\|_{2}^{2} .
\end{aligned}
$$

Thus, $|f(x)-f(y)| \leq \sqrt{2 \pi n}\|x-y\|_{2}$, and appealing to Theorem 6 with $\sigma=\sqrt{2 \pi n}$ yields

$$
\mathbb{P}\left(\|A X\|_{1}>\mathbb{E}\left[\|A X\|_{1}\right]+t n\right)=\mathbb{P}(F>\mathbb{E}[F]+t n) \leq e^{-2(n t)^{2} /(\pi \sqrt{2 \pi n})^{2}}=e^{-n t^{2} / \pi^{3}} .
$$

Finishing the proof for $\mathbb{K}=\mathbb{C}$

Proposition 8. Let $\|A\|_{2} \leq 1$ and $X \in \mathbb{C}^{n}$ be as in Section 国. If $\mathbb{E}\left[\|A X\|_{1} / n\right]=\mu$, then

$$
\mathbb{E}\left[\left(\|A X\|_{1} / n\right)^{n}\right] \leq 2 \exp \left[-3 n(1-\mu)^{2} / 100\right] .
$$

Proof. Let $L=t \mu+(1-t)$ with $t \in[0,1]$ to be determined. Since $0 \leq\|A X\|_{1} / n \leq 1$, we have (appealing to Proposition 7 for the last inequality)

$$
\begin{aligned}
\mathbb{E}\left[\left(\|A X\|_{1} / n\right)^{n}\right] & \leq L^{n}+\mathbb{P}\left(\|A X\|_{1} / n>L\right) \\
& \leq \exp [-n(1-L)]+\mathbb{P}\left(\|A X\|_{1} / n-\mu>(1-t)(1-\mu)\right) \\
& \leq \exp [-n t(1-\mu)]+\exp \left[-n(1-t)^{2}(1-\mu)^{2} / \pi^{3}\right],
\end{aligned}
$$

We now take $2 t(1-\mu)=\pi^{3}+2-2 \mu-\pi^{3 / 2} \sqrt{\pi^{3}+4-4 \mu}$ (for which $t$ does lie in the interval $[0,1]$ ), so as to make the exponents equal. For this $t$, we obtain

$$
\mathbb{E}\left[\left(\|A X\|_{1} / n\right)^{n}\right] \leq 2 \exp \left[-n\left(2 \mu+\pi^{3 / 2} \sqrt{\pi^{3}+4-4 \mu}-\pi^{3}-2\right) / 2\right] .
$$

Then appealing to the Taylor series at $\mu=1$, we see that for all $\mu \in[0,1]$,

$$
\frac{2 \mu+\pi^{3 / 2} \sqrt{\pi^{3}+4-4 \mu}-\pi^{3}-2}{2} \geq \frac{(1-\mu)^{2}}{\pi^{3}}-\frac{2(1-\mu)^{3}}{\pi^{6}} \geq(1-\mu)^{2}\left(\frac{1}{\pi^{3}}-\frac{2}{\pi^{6}}\right) \geq \frac{3(1-\mu)^{2}}{100}
$$

We then readily obtain Theorem 2 simply by combining Propositions 5 and 8 and using the fact that if $\|A\|_{2} \leq 1$, then $0 \leq h_{\infty}(A) \leq h_{2}(A) \leq 1$.

\section{Proof of Theorem 3 (better results for $\mathbb{K}=\mathbb{R}$ )}

For matrices over $\mathbb{R}$, our general strategy is the same as before, but we first partition the rows of $A$ into those that contain 'big' entries and those that do not. We show that the contribution due to rows with large entries has small variance, and although the rows without large entries may each contribute something of high variance, we benefit from the fact that there simply aren't that many such rows. In this way, we are able to obtain better concentration of $\|A X\|_{1}$ about its mean, which in turn gives a better bound on $\operatorname{per}(A)$. 
We are not sure exactly how to adapt this argument when $\mathbb{K}=\mathbb{C}$, although we admittedly didn't try very hard to do so. We feel confident (especially in light of Theorem (3) that Theorem 2 can be improved, but we do not think that Theorem 3 is best possible either (which is why we haven't worried so much about extending it to $\mathbb{K}=\mathbb{C}$ ). See Section 5 for a discussion of several related conjectures (some perhaps more true than others) and open problems.

\section{Set-up for the real-valued case}

As in Section 2, we let $A$ be an $n \times n$ matrix over $\mathbb{R}$ with $\|A\|_{2} \leq 1$. Define $t=1-h_{\infty}(A)$. Then to prove Theorem 3 , our goal is to show

$$
|\operatorname{per}(A)| \leq(n+6) \exp [-\sqrt{n t} / 400]
$$

Let $\varepsilon>0$ and $1 / 10>\lambda>0$ be parameters to be determined (we will end up choosing $\varepsilon=t / 10$ and $\lambda=64 / \sqrt{n t}$ ). We now partition the rows of $A$ into "big rows" (those containing an element of absolute value at least $1-\lambda$ ) and "small rows" (the rest). Suppose there are $b$ big rows and $l=n-b$ small rows. Recall that because $\|A\|_{2} \leq 1$, each row and column of $A$ has $l_{2}$-norm at most 1. Thus, 'large' entries (those of absolute value at least $1-\lambda$ ) must appear in different rows and columns. By multiplying $A$ by appropriate permutation matrices and the appropriate \pm 1 -diagonal matrix (which changes neither the norm, nor the absolute value of the permanent, nor the values of $t, b$, or $l$ ), we can assume $A$ is of the form:

$$
A=\left(\begin{array}{c}
B \\
L
\end{array}\right)
$$

where $B$ is a $b \times n$ matrix, the $(i, i)$-entries of $B$ are all positive with size at least $1-\lambda$, and all the rest of the entries in $A$ have absolute value less than $1-\lambda$. For convenience, we will assume $b>0$ and $l>0$, for if not, our same argument would apply with only superficial alterations.

We recall our earlier set-up as in the complex-case (but with $X \in \mathbb{R}^{n}$ now uniformly distributed over $\left.\{-1,1\}^{n}\right)$. Then for all $\tilde{\mu}_{B}, \tilde{\mu}_{L} \geq 0$, we have

$$
\begin{aligned}
|\operatorname{per}(A)| & \leq \mathbb{E}_{X}\left[\left(\frac{\|A X\|_{1}}{n}\right)^{n}\right]=\mathbb{E}_{X}\left[\left(\frac{\|L X\|_{1}+\|B X\|_{1}}{n}\right)^{n}\right] \\
& \leq\left(\frac{\tilde{\mu}_{L}+\tilde{\mu}_{B}}{n}+2 \varepsilon\right)^{n}+\mathbb{P}\left(\|L X\|_{1} \geq \tilde{\mu}_{L}+\varepsilon n\right)+\mathbb{P}\left(\|B X\|_{1} \geq \tilde{\mu}_{B}+\varepsilon n\right),
\end{aligned}
$$

where (as before) the last inequality is justified by the fact that the random variable within the expected value is bounded above by 1 .

We choose

$$
\begin{aligned}
& \tilde{\mu}_{B}=\sum_{i=1}^{b}\left[\sqrt{\frac{2}{\pi}}+\left(1-\sqrt{\frac{2}{\pi}}\right)\left\|r_{i}\right\|_{\infty}\right]=\sum_{i=1}^{b}\left[1-\left(1-\sqrt{\frac{2}{\pi}}\right)\left(1-\left\|r_{i}\right\|_{\infty}\right)\right], \quad \text { and } \\
& \tilde{\mu}_{L}=\sum_{i>b}^{n}\left[\sqrt{\frac{2}{\pi}}+\left(1-\sqrt{\frac{2}{\pi}}\right)\left\|r_{i}\right\|_{\infty}\right]=\sum_{i>b}^{n}\left[1-\left(1-\sqrt{\frac{2}{\pi}}\right)\left(1-\left\|r_{i}\right\|_{\infty}\right)\right]
\end{aligned}
$$

where (again) $r_{i}$ is the $i^{\text {th }}$ row of $A$ (note, $\left\|r_{i}\right\|_{\infty}=b_{i, i}$ for all $i \leq b$ ). Then by Theorem 4 (this time with $\mathbb{K}=\mathbb{R})$, we have $\tilde{\mu}_{L} \geq \mathbb{E}\left[\|L X\|_{1}\right]$ and $\tilde{\mu}_{B} \geq \mathbb{E}\left[\|B X\|_{1}\right]$, and by the definitions

$$
\frac{\tilde{\mu}_{L}+\tilde{\mu}_{B}}{n}=1-\left(1-\sqrt{\frac{2}{\pi}}\right) \frac{1}{n} \sum_{i=1}^{n}\left(1-\left\|r_{i}\right\|_{\infty}\right)=1-\left(1-\sqrt{\frac{2}{\pi}}\right) t .
$$


To take advantage of (1), we need only exhibit concentration bounds for $\|L X\|_{1}$ and $\|B X\|_{1}$.

\section{Concentration of $\|L X\|_{1}$}

To show concentration of $\|L X\|_{1}$ about its mean, we will again apply a version of Talagrand's inequality (but this time suited for the discrete distribution over $\{-1,1\}^{n}$ ). Instead of showing the derivation of this from the corresponding general result in [9] (as we did before), we will simply cite [3], in which the following statement appears as Theorem 3.3.

Theorem 9. Suppose $M$ is a $k \times n$ real-valued matrix such that $\|M \vec{x}\|_{1} \leq \sigma\|\vec{x}\|_{2}$ for all $\vec{x} \in \mathbb{R}^{n}$. Let $\xi \in \mathbb{R}^{n}$ be chosen uniformly from $\{-1,1\}^{n}$, and let $m$ be a median of $\|M \xi\|_{1}$. Then for all $\gamma \geq 0$, we have $\mathbb{P}\left(||\left|M \xi \|_{1}-m\right|>\gamma\right) \leq 4 e^{-\gamma^{2} /\left(8 \sigma^{2}\right)}$.

Lemma 10. With notation as before, if $\varepsilon n \geq 16 \sqrt{n t \log (n) / \lambda}$, then

$$
\mathbb{P}\left(\|L X\|_{1} \geq \tilde{\mu}_{L}+\varepsilon n\right) \leq 4 \exp \left[\frac{-\varepsilon^{2} n \lambda}{32 t}\right] .
$$

Proof. Note that for all $\vec{x} \in \mathbb{R}^{n}$, we have $\|L \vec{x}\|_{1} \leq \sqrt{l}\|L \vec{x}\|_{2} \leq \sqrt{l}\|A \vec{x}\|_{2} \leq \sqrt{l}\|\vec{x}\|_{2}$. Thus, if $m$ is a median of $\|L X\|_{1}$, then by Theorem 9, we have

$$
\mathbb{P}\left(||\left|L X \|_{1}-m\right|>\gamma\right) \leq 4 e^{-\gamma^{2} /(8 l)} .
$$

From this, we see that $\|L X\|_{1}$ is tightly concentrated about its median. However, this also implies

$$
m \leq \mathbb{E}\left[\|L X\|_{1}\right]+8 \sqrt{l \log n},
$$

since otherwise, we would have

$$
\begin{aligned}
\mathbb{E}\left[\|L X\|_{1}\right] & \geq\left(\mathbb{E}\left[\|L X\|_{1}\right]+4 \sqrt{l \log n}\right) \cdot \mathbb{P}\left(\left|\|L X\|_{1}-m\right| \leq 4 \sqrt{l \log n}\right) \\
& \geq\left(\mathbb{E}\left[\|L X\|_{1}\right]+4 \sqrt{l \log n}\right) \cdot\left(1-4 / n^{2}\right) \\
& =\mathbb{E}\left[\|L X\|_{1}\right]+4 \sqrt{l \log n}-\left(\mathbb{E}\left[\|L X\|_{1}\right]+4 \sqrt{l \log n}\right) \cdot 4 / n^{2}
\end{aligned}
$$

And subtracting $\mathbb{E}\left[\|L X\|_{1}\right]$ from both sides and rearranging, we would obtain

$$
n^{2} \leq 4+\frac{\mathbb{E}\left[\|L X\|_{1}\right]}{\sqrt{l \log n}} \leq 4+\frac{n}{\sqrt{\log n}}
$$

which is a contradiction if $n>2$ (whereas for $n \leq 2$, the desired bound on $m$ is implied by $m \leq n$ [not that it matters]). Therefore, appealing to (4), we have

$$
\mathbb{P}\left(\|L X\|_{1} \geq \tilde{\mu}_{L}+\varepsilon n\right) \leq \mathbb{P}\left(\|L X\|_{1} \geq \mathbb{E}\left[\|L X\|_{1}\right]+\varepsilon n\right) \leq \mathbb{P}\left(\|L X\|_{1} \geq m+\varepsilon n-8 \sqrt{l \log n}\right) .
$$

Furthermore, if $\varepsilon n \geq 16 \sqrt{l \log n}$, then we can combine this with (3) to obtain

$$
\text { if } \varepsilon n \geq 16 \sqrt{l \log n} \text {, then } \quad \mathbb{P}\left(\|L X\|_{1} \geq \tilde{\mu}_{L}+\varepsilon n\right) \leq 4 \exp \left[\frac{-\varepsilon^{2} n^{2}}{32 l}\right] .
$$

Finally, since $n t \geq \sum_{i=b+1}^{n}\left(1-\left\|r_{i}\right\|_{\infty}\right) \geq l \lambda$, we know $l \leq n t / \lambda$, completing the proof by (5).

\section{Concentration of $\|B X\|_{1}$}

We now focus on getting an upper bound on $\mathbb{P}\left(\|B X\|_{1} \geq \tilde{\mu}_{B}+\varepsilon n\right)$. We first recall the following classical concentration result. 
Proposition 11 (Hoeffding's inequality). Let $a_{1}, \ldots, a_{k}$ be real numbers (not all of which are 0), and let $\xi_{1}, \xi_{2}, \ldots, \xi_{k}$ be independent each distributed uniformly on $\{-1,1\}$. Then for all $\gamma \geq 0$,

$$
\mathbb{P}\left(\sum_{i=1}^{k} a_{i} \xi_{i} \geq \gamma\right) \leq \exp \left[\frac{-\gamma^{2}}{2 \sum_{i=1}^{k} a_{i}^{2}}\right]
$$

Let $\tilde{B}=\left(\begin{array}{c}B \\ 0\end{array}\right)$ be the $n \times n$ matrix whose first $b$ rows are given by $B$ and the rest are 0 . Our key step here is replacing $\|B X\|_{1}$ with $\langle X, \tilde{B} X\rangle$, via the following lemma1.

Lemma 12. With notation as before, if $\lambda<0.1$ then

$$
\mathbb{P}\left(\|B X\|_{1} \geq \tilde{\mu}_{B}+\varepsilon n\right) \leq \mathbb{P}\left(\langle X, \tilde{B} X\rangle \geq \tilde{\mu}_{B}+\varepsilon n\right)+n e^{-1 /(5 \lambda)} .
$$

Proof. It suffices to show $\mathbb{P}\left(\|B X\|_{1} \neq\langle X, \tilde{B} X\rangle\right) \leq n e^{-1 /(5 \lambda)}$. The idea is that since each row of $B$ is dominated by a single large entry (namely $b_{i, i}$ ), each entry of $B X$ is a random sum dominated by a single large term (namely $X_{i} b_{i, i}$ ). Thus, it is very unlikely that any entry of $B X$ would have a different sign than $X_{i} b_{i, i}$. This is made rigorous as follows.

Recall that we ordered the columns of $B$ so that the $(i, i)$-entry is the largest in its row, and that $b_{i, i} \geq 1-\lambda$. Letting $Y_{i}$ be the $i^{\text {th }}$ coordinate of $B X$, we have, by a simple union bound,

$$
\mathbb{P}\left(\|B X\|_{1} \neq\langle X, \tilde{B} X\rangle\right) \leq \sum_{i=1}^{b} \mathbb{P}\left(\left|Y_{i}\right| \neq X_{i} Y_{i}\right)=\sum_{i=1}^{b} \mathbb{P}\left(X_{i} Y_{i}<0\right)=\sum_{i=1}^{b} \mathbb{P}\left(\sum_{j=1}^{n} X_{i} X_{j} b_{i, j}<0\right) .
$$

Using the fact that for any given $i$, the random vector $\left(X_{i} X_{j}\right)_{j \neq i}$ has the same joint distribution as $\left(X_{j}\right)_{j \neq i}\left(\right.$ and that $X_{i}^{2}=1$ ), we obtain by Proposition 11

$$
\sum_{i=1}^{b} \mathbb{P}\left(\sum_{j=1}^{n} X_{i} X_{j} b_{i, j}<0\right)=\sum_{i=1}^{b} \mathbb{P}\left(b_{i, i}<\sum_{j \neq i}^{n} X_{j} b_{i, j}\right) \leq \sum_{i=1}^{b} \exp \left[\frac{-b_{i, i}^{2}}{2 \sum_{i \neq j} b_{i, j}^{2}}\right] .
$$

Since $b_{i, i} \geq 1-\lambda$ and $\sum_{j} b_{i, j}^{2} \leq 1$, this in turn is bounded by

$$
\sum_{i=1}^{b} \exp \left[\frac{-b_{i, i}^{2}}{2 \sum_{i \neq j} b_{i, j}^{2}}\right] \leq n \exp \left[\frac{-(1-\lambda)^{2}}{2\left(1-(1-\lambda)^{2}\right)}\right] \leq n e^{-1 /(5 \lambda)},
$$

where the last inequality is justified because $0<\lambda<0.1$.

We can now exploit the fact that $\langle X, \tilde{B} X\rangle$ is a degree two polynomial over $\{-1,1\}^{n}$, allowing us to use any of a variety of concentration inequalities. We will use an inequality of Bonami [4, which was the first hypercontractivity inequality of its type. A detailed exposition of such results can be found in chapter 9 of O'Donnell's book [11, and a comparison of this to more recent polynomial concentration inequalities can be found in [12].

Theorem 13 (Bonami 4, 1970). Let $F: \mathbb{R}^{n} \rightarrow \mathbb{R}$ be a degree $k$ polynomial, and consider the random variable $Z=F\left(\xi_{1}, \xi_{2}, \ldots, \xi_{n}\right)$, where the $\xi_{i}$ are independent with each distributed uniformly over $\{-1,1\}$. Then for all $q \geq 2$, we have $\mathbb{E}\left[|Z|^{q}\right] \leq\left((q-1)^{k} \mathbb{E}\left[Z^{2}\right]\right)^{q / 2}$.

Lemma 14. With notation as before, if $\varepsilon n \geq 4 e \sqrt{n t}$, then

$$
\mathbb{P}\left(\langle X, \tilde{B} X\rangle \geq \tilde{\mu}_{B}+\varepsilon n\right) \leq \exp \left(\frac{-\varepsilon n}{2 e \sqrt{n t}}\right)
$$

\footnotetext{
${ }^{1}$ Extending this step is the main obstacle to applying the present argument when $\mathbb{K}=\mathbb{C}$.
} 
Proof. For $\vec{x} \in \mathbb{R}^{n}$, define $F\left(x_{1}, x_{2}, \ldots, x_{n}\right)=\langle\vec{x}, \tilde{B} \vec{x}\rangle-\sum_{i=1}^{b} b_{i, i}$, and define the random variable

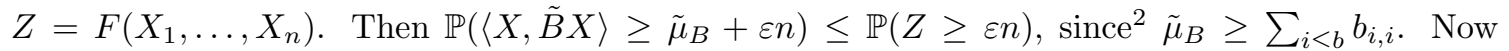
$F\left(x_{1}, x_{2}, \ldots, x_{n}\right)$ is a degree 2 polynomial, and moreover, by expanding out the sums and using the fact that terms such as $\mathbb{E}\left[X_{i} X_{j}\right]$ vanish when $i \neq j$, we obtain

$$
\begin{aligned}
\mathbb{E}\left[Z^{2}\right] & =\mathbb{E}\left[\left(\sum_{i=1}^{b}\left[-b_{i, i}+\sum_{j=1}^{b} X_{i} X_{j} b_{i, j}\right]+\sum_{i=1}^{b} \sum_{j=b+1}^{n} X_{i} X_{j} b_{i, j}\right)^{2}\right] \\
& =\mathbb{E}\left[\left(\sum_{i=1}^{b}\left[-b_{i, i}+\sum_{j=1}^{b} X_{i} X_{j} b_{i, j}\right]\right)^{2}\right]+\mathbb{E}\left[\left(\sum_{i=1}^{b} \sum_{j=b+1}^{n} X_{i} X_{j} b_{i, j}\right)^{2}\right] \\
& =\sum_{i=1}^{b} \sum_{j<i}\left(b_{i, j}+b_{j, i}\right)^{2}+\sum_{i=1}^{b} \sum_{j=b+1}^{n} b_{i, j}^{2} \leq 2 \sum_{i=1}^{b} \sum_{j<i}\left(b_{i, j}^{2}+b_{j, i}^{2}\right)+2 \sum_{i=1}^{b} \sum_{j=b+1}^{n} b_{i, j}^{2} \\
& =2 \sum_{i=1}^{b}\left(-b_{i, i}^{2}+\sum_{j=1}^{n} b_{i, j}^{2}\right) \leq 2 \sum_{i=1}^{b}\left(1-b_{i, i}^{2}\right) \leq 4 \sum_{i=1}^{b}\left(1-b_{i, i}\right) \leq 4 n t .
\end{aligned}
$$

Applying Theorem 13 with $q=\varepsilon n /(2 e \sqrt{n t})$ - which is valid since by hypothesis this ratio is at least 2 -together with Markov's inequality, we obtain

$$
\mathbb{P}(Z \geq \varepsilon n) \leq \mathbb{P}\left(|Z|^{q} \geq(\varepsilon n)^{q}\right) \leq \frac{\mathbb{E}\left[|Z|^{q}\right]}{(\varepsilon n)^{q}} \leq\left(\frac{(q-1) 2 \sqrt{n t}}{\varepsilon n}\right)^{q} \leq \exp \left(\frac{-\varepsilon n}{2 e \sqrt{n t}}\right) .
$$

\section{Finishing the proof for $\mathbb{K}=\mathbb{R}$}

We now need to pick $\varepsilon$ and $\lambda$ to optimize the tradeoffs between our various upper bounds. We need the assumptions of Lemmas 10, 12, and 14-namely (i) $\varepsilon n \geq 16 \sqrt{n t \log (n) / \lambda}$, (ii) $\lambda<0.1$, and (iii) $\varepsilon n \geq 4 e \sqrt{n t}$-in which case we can combine these lemmas with (11) and (2) to obtain

$$
\begin{aligned}
|\operatorname{per}(A)| & \leq\left(2 \varepsilon+\frac{\tilde{\mu}_{L}+\tilde{\mu}_{B}}{n}\right)^{n}+\mathbb{P}\left(\|L X\|_{1} \geq \tilde{\mu}_{L}+\varepsilon n\right)+\mathbb{P}\left(\|B X\|_{1} \geq \tilde{\mu}_{B}+\varepsilon n\right) \\
& \leq\left(2 \varepsilon+1-\left(1-\sqrt{\frac{2}{\pi}}\right) t\right)^{n}+4 \exp \left[\frac{-\varepsilon^{2} n \lambda}{32 t}\right]+n e^{-1 /(5 \lambda)}+\exp \left(\frac{-\varepsilon n}{2 e \sqrt{n t}}\right) .
\end{aligned}
$$

We will take $\varepsilon=t / 10$ and $\lambda=64 / \sqrt{n t}$, for which we claim that conditions (i), (ii), and (iii) are satisfied. Note that since our goal is to show $|\operatorname{per}(A)| \leq(n+6) \exp [-\sqrt{n t} / 400]$, we may assume $\sqrt{n t} / \log (n+6) \geq 400$ (or the bound we are trying for is worse than the trivial bound of 1 ) (of course, in any case we are really more interested in large $n$ ). Notice that with $\varepsilon$ and $\lambda$ as above:

(i) $\varepsilon n \geq 16 \sqrt{n t \log (n) / \lambda}$ is equivalent to $\sqrt{n t} \geq 400 \log n$;

(ii) $\lambda<0.1$ is equivalent to $\sqrt{n t}>640$; and

(iii) $\varepsilon n \geq 4 e \sqrt{n t}$ is equivalent to $\sqrt{n t} \geq 40 e$.

\footnotetext{
${ }^{2}$ In fact, we could have simply taken $\tilde{\mu}_{B}=\sum_{i \leq b} b_{i, i}$, but we chose instead to define it similarly to $\tilde{\mu}_{L}$, a change which only affects the constants in our end result.
} 
Thus, these choices of $\lambda$ and $\varepsilon$ allow us to appeal to the aforementioned results, obtaining

$$
\begin{aligned}
|\operatorname{per}(A)| & \leq\left(2 \varepsilon+1-\left(1-\sqrt{\frac{2}{\pi}}\right) t\right)^{n}+4 \exp \left[\frac{-\varepsilon^{2} n \lambda}{32 t}\right]+n e^{-1 /(5 \lambda)}+\exp \left(\frac{-\varepsilon n}{2 e \sqrt{n t}}\right) \\
& \leq \exp [-n t(1-\sqrt{2 / \pi}-0.2)]+4 \exp \left[\frac{-\sqrt{n t}}{50}\right]+n \exp \left[-\frac{\sqrt{n t}}{320}\right]+\exp \left[\frac{-\sqrt{n t}}{20 e}\right] \\
& \leq(n+6) \exp \left[\frac{-\sqrt{n t}}{400}\right],
\end{aligned}
$$

which completes the proof of Theorem 3 .

\section{Conclusion}

Our biggest (and most natural) open question concerns the optimality of our main results. Namely, a proof of Conjecture 1 as stated in Section 1 would be very interesting. The main barrier preventing us from proving this conjecture is our reliance on Talagrand's inequality. For $\mathbb{K}=\mathbb{R}$, we partially mitigated the cost of using this inequality via Lemma 10, but the application of Theorem 9 was still a crucial (though not the only) bottleneck. Our argument could conceivably be pushed further either by a more careful analysis that better uses (5) or by a more nuanced argument that splits the matrix $A$ into more than two pieces.

One could also try to avoid using Talagrand's inequality altogether. It is possible that some stronger inequality could replace it (by taking advantage of some aspects particular to our situation), but a more likely "quick fix" of this sort would be a more direct estimate of $\mathbb{E}\left[\left(\|A X\|_{1} / n\right)^{n}\right]$ (in the real case, $A X$ is simply a vector-valued Rademacher sum, which is a well-studied random variable). On the other hand, it could be that the convexity bounds on the Glynn estimator already give away too much to recover anything stronger than what we have.

An entirely different approach would be to determine among matrices with given norm and $h_{\infty}$, which ones maximize $|\operatorname{per}(A)|$ (it does not seem impossible that this maximum is always attained by a circulant matrix with all real entries). A characterization of these extremal matrices would certainly be very appealing, and one might hope that thinking along these lines would suggest a more combinatorial approach.

As far as Question B is concerned, we feel that there is still more to be said beyond the present results. Namely, our results only provide a necessary condition for a matrix to have a large permanent (i.e., $h_{\infty}$ must be large). But there is no clean converse to this statement; consider for example a diagonal matrix with most of its diagonal entries equal to 1 except for one of them equal to 0 (this has large $h_{\infty}$ and permanent 0 ). To continue the spirit of the question, we state the following variation of Question B (essentially echoing a question of [1]):

Problem B': Find a (deterministic) polynomial-time algorithm that takes an $n \times n$ matrix $A$ of norm 1 and decides whether $|\operatorname{per}(A)|<n^{-100}$ or $|\operatorname{per}(A)|>n^{-10}$ (with the understanding that the input matrix will satisfy one of these inequalities).

We attempted this along the following lines: "if the matrix has large permanent, it must have many rows each of which is dominated by a single large entry. If the matrix is of this form, then [heuristic] hopefully that means the permanent is dominated by terms that use at least most of these large entries. Since there are so many large entries, we can efficiently compute the exact contribution of these dominant terms." However, our current results do not allow us to conclude that there are enough rows with large entries (we would like all but about $\log n$ of the rows but are limited to all but about $\log ^{2} n$ when $\mathbb{K}=\mathbb{R}$ and $\sqrt{n \log n}$ when $\mathbb{K}=\mathbb{C}$ ). And in fact, even if we 
could improve our result to the conjectured (and best possible) bound mentioned above, we still do not quite see how to make this heuristic argument yield a polynomial-time algorithm. We should note that Gurvits [6] found a randomized algorithm accomplishing the goal of Problem $\mathrm{B}^{\prime}$, and in the deterministic setting, progress towards Problem $\mathrm{B}^{\prime}$ was made in 1 which gives an algorithm in the case that the entries of $A$ are non-negative.

\section{Further remarks}

- We note that there is a lot of freedom in choosing the random variable $X \in \mathbb{K}^{n}$ for the Glynn estimator ( $X$ just needs to have independent components each satisfying $\mathbb{E}\left[X_{i}\right]=0$ and $\left.\mathbb{E}\left[\left|X_{i}\right|^{2}\right]=1\right)$. For example, when $\mathbb{K}=\mathbb{R}$, it is tempting to replace $X \in \mathbb{R}^{n}$ with an $n$-dimensional Gaussian and bound the Glynn estimator by something like

$$
|\operatorname{per}(A)|=\left|\mathbb{E}\left[\prod_{i} X_{i} Y_{i}\right]\right| \leq \mathbb{E}\left[\prod_{i}\left|X_{i} Y_{i}\right|\right] \leq \mathbb{E}\left[\left(\frac{1}{n} \sum_{i}\left|X_{i} Y_{i}\right|\right)^{n}\right] .
$$

But even if $A$ is the identity matrix this is already (exponentially) larger than 1, which illustrates the difficulty with this approach.

- Via an entirely different method, we were also able to get an upper bound on the permanent for matrices having only non-negative real entries by appealing to the results of [7]. Unfortunately, the bound we obtained is strictly weaker than the results of the present paper, so it is omitted.

Acknowledgement: We thank Hoi Nguyen for introducing us to this problem and sharing [10].

\section{References}

[1] Scott Aaronson and Travis Hance. Generalizing and derandomizing Gurvits's approximation algorithm for the permanent. Quantum Inf. Comput., 14(7-8):541-559, 2014.

[2] Scott Aaronson and Hoi Nguyen. Near invariance of the hypercube. Israel Journal of Mathematics, 2016.

[3] Nir Ailon and Edo Liberty. Fast dimension reduction using Rademacher series on dual BCH codes. Discrete Comput. Geom., 42(4):615-630, 2009.

[4] Aline Bonami. Étude des coefficients de Fourier des fonctions de $L^{p}(G)$. Ann. Inst. Fourier (Grenoble), 20(fasc. 2):335-402 (1971), 1970.

[5] David G. Glynn. The permanent of a square matrix. European J. Combin., 31(7):1887-1891, 2010.

[6] Leonid Gurvits. On the complexity of mixed discriminants and related problems. In Mathematical Foundations of Computer Science 2005, pages 447-458. Springer, 2005.

[7] Leonid Gurvits and Alex Samorodnitsky. Bounds on the permanent and some applications. In 2014 IEEE 55th Annual Symposium on Foundations of Computer Science (FOCS), pages 90-99. IEEE, 2014.

[8] Hermann König, Carsten Schütt, and Nicole Tomczak-Jaegermann. Projection constants of symmetric spaces and variants of Khintchine's inequality. J. Reine Angew. Math., 511:1-42, 1999.

[9] Michel Ledoux and Michel Talagrand. Probability in Banach spaces. Classics in Mathematics. Springer-Verlag, Berlin, 2011. Isoperimetry and processes, Reprint of the 1991 edition.

[10] Hoi Nguyen. On matrices of large permanent. Private communication, 2016. 
[11] Ryan O'Donnell. Analysis of boolean functions. Cambridge University Press, 2014.

[12] Warren Schudy and Maxim Sviridenko. Concentration and moment inequalities for polynomials of independent random variables. In Proceedings of the Twenty-Third Annual ACM-SIAM Symposium on Discrete Algorithms, pages 437-446. ACM, New York, 2012.

[13] L. G. Valiant. The complexity of computing the permanent. Theoret. Comput. Sci., 8(2):189201, 1979.

Department of Mathematics

Rutgers University

Piscataway, NJ 08854

rkb730math.rutgers.edu

prd41@math.rutgers.edu 